\title{
Optimización de la producción forestal maderable y conservación del ecosistema en bosques comunitarios en el sur de México
}

\author{
Optimization of timber production and ecosystem conservation \\ in community forests of southern Mexico
}

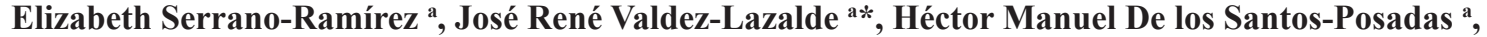 \\ Román Anselmo Mora-Gutíerrez ${ }^{\text {b }}$, Gregorio Ángeles-Pérez ${ }^{\text {a }}$

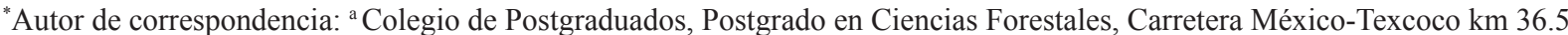 \\ Montecillo, Estado de México, México, CP. 56230, tel.: 52-595-9520246, valdez@colpos.mx \\ ${ }^{\mathrm{b}}$ Universidad Autónoma Metropolitana, Posgrado en Optimización, Ciudad de México, México.
}

\begin{abstract}
SUMMARY
Current forest management approaches require management strategies that identify where and when to carry out harvest activities that satisfy the demand for wood products while forest ecosystem services are conserved. This work makes the TRIAD zoning approach operational through a mathematical model to optimize the forestry planning process. This approach proposes dividing the forest under management into an intensive harvesting area, an extensive harvesting area, and a conservation area, according to the production objectives established. A mixed integer nonlinear optimization model was formulated to maximize the present value of gains derived from timber production, while satisficing operative and biodiversity conservation restrictions associated with the forest community of San Pedro El Alto, Oaxaca, Mexico. The model was solved through a genetic algorithm, in their simple version (GNS), and two variants of it (GN2 and GN3). The process allowed completing three objectives: 1) to propose and validate a model capable of generating comparable solutions to the current management scenario in the community, 2) to compare three different solution algorithms, and 3) to offer an efficient algorithm to solve similar optimization problems.
\end{abstract}

Key words: biodiversity conservation, forest management planning, genetic algorithms, functional zoning, TRIAD.

\section{RESUMEN}

El enfoque actual de gestión forestal requiere de estrategias de manejo que permitan identificar dónde y cuándo ejecutar actividades silvícolas, con objeto de satisfacer la demanda de productos de madera mientras se conservan los servicios que el ecosistema proporciona. El presente trabajo hace operacional el enfoque de zonificación TRIAD a través de un modelo matemático para optimizar el proceso de planificación del aprovechamiento forestal. Este enfoque propone dividir el territorio forestal bajo gestión en una zona de aprovechamiento intensivo, una zona de aprovechamiento extensivo y una zona destinada a la conservación del ecosistema; cada una designada en función de los objetivos de producción establecidos. Se formuló un modelo no lineal entero mixto para maximizar el ingreso derivado del aprovechamiento forestal maderable, en términos de valor presente, y al mismo tiempo satisfacer restricciones operativas y de conservación asociadas al bosque comunitario de San Pedro El Alto, Oaxaca, México. El modelo fue resuelto mediante la implementación de un algoritmo genético, en su versión simple (GNS) y dos variantes de la misma (GN2 y GN3). El proceso permitió cumplimentar tres objetivos: 1) proponer y validar un modelo capaz de generar soluciones comparables con el escenario actual de manejo en la comunidad, 2) comparar tres diferentes algoritmos de solución y 3) ofrecer un algoritmo eficiente para resolver problemas de optimización similares.

Palabras clave: conservación de biodiversidad, manejo forestal, algoritmos genéticos, zonificación funcional, TRIAD.

\section{INTRODUCCIÓN}

La gestión sostenible de los recursos naturales, en general, involucra decidir la estrategia más adecuada para el aprovechamiento y cuidado de los mismos. En particular, gestionar el aprovechamiento y cultivo de los bosques implica decidir cuándo y dónde cosechar para satisfacer la demanda de productos forestales y al mismo tiempo cum- plir con las políticas sociales de conservación; implica encontrar estrategias de equilibrio entre las demandas de la industria forestal y otros valores ecológicos de interés para la sociedad (Tittler et al. 2015, Vergara et al. 2015). En el pasado, las estrategias de aprovechamiento de los bosques se enfocaban a maximizar el ingreso en un horizonte de planeación (Bouchard et al. 2017). Actualmente, uno de los objetivos de la gestión forestal es la conservación del 
ecosistema, además de la provisión de otros servicios ecosistémicos.

El reto de los administradores de recursos forestales consiste en determinar cuál es la cantidad adecuada de cada producto o servicio que permita maximizar la utilidad del bosque (Marušák et al. 2015). Para esta tarea se utilizan modelos de optimización, ya sean de programación lineal o entera mixta (Hof et al. 1994) o técnicas heurísticas (Murray y Church 1995), que facilitan y respaldan la toma de decisiones referente a los regímenes silvícolas aplicables en un bosque, el volumen maderable a cosechar en un tiempo determinado, la edad óptima para la alternancia en el uso de tierras, las áreas potenciales de producción, etc. Estos modelos no solo son capaces de optimizar uno o más objetivos de gestión, también pueden satisfacer una gran variedad de restricciones financieras y ecológicas (Quintero et al. 2010, Hoganson y Meyer 2015).

Un enfoque de planeación que pretende lograr este tipo de equilibrio es la zonificación forestal funcional (enfoque TRIAD) (Seymour y Hunter 1992), el cual implica asignar áreas forestales con base en los diferentes productos o servicios posibles a obtener. Se parte del supuesto que la especialización en el uso del bosque (zonificación), podría evitar que se asignen ineficientemente los recursos necesarios para producir beneficios maderables y no maderables, pues gestionar el uso múltiple en una sola área implica un proceso ineficiente para obtener los diferentes productos al mismo tiempo (Côté et al. 2010, Tittler et al. 2015).

En México, el empleo de modelos de optimización financiera en la planificación del aprovechamiento de los bosques es limitado, casi inexistente. La mayoría de los planes de manejo forestal se elaboran con base en la pericia de los administradores forestales, fundamentando el proceso de planificación en un análisis de las existencias maderables, la posibilidad de cosecha considerando el incremento del bosque, y en la definición de una meta de cosecha anual (volumen meta), no necesariamente igual a la posibilidad. Criterios de operación local y rasgos culturales de las comunidades forestales, comúnmente influyen para que el volumen real de cosecha se defina por debajo de la posibilidad. El resultado son planes de manejo de eficiencia económica desconocida. Por tal motivo, el presente trabajo propone y resuelve, mediante algoritmos genéticos, un modelo no lineal entero mixto (PNLEM), que optimiza el ingreso económico, producto de la cosecha de madera, considerando restricciones operativas y de conservación del ecosistema bajo un enfoque de zonificación TRIAD. El modelo asigna una sección del bosque a la zona de aprovechamiento intensivo para la producción de madera, otra sección a la zona de aprovechamiento extensivo para la obtención de productos no maderables y cosecha de madera, pero en una escala menor; y la superficie restante a una zona de conservación para proteger la biodiversidad y la funcionalidad del ecosistema. Paralelamente se realiza un análisis comparativo de tres algoritmos de solución al modelo matemático planteado.

\section{MÉTODOS}

Datos. El PNLEM se desarrolló para el área forestal bajo manejo de la comunidad San Pedro El Alto, Zimatlán, ubicada en el estado de Oaxaca, sur de México. Se tomó como base el Plan de Manejo Forestal (PMF) 2016-2017/ 2025-2026 de la comunidad, el cual comprende 8.427 ha, divididas en 556 rodales (unidades mínimas de manejo), y contiene información por rodal correspondiente a: volumen maderable disponible por hectárea $\left(\mathrm{m}^{3} / \mathrm{ha}\right)$, calidad del sitio, incremento medio anual del volumen maderable $\left(\mathrm{m}^{3} / \mathrm{ha}\right)$, tipo de hábitat, potencial de aprovechamiento y tratamiento silvícola programado. El modelo de optimización de la producción bajo el enfoque TRIAD considera aprovechamiento (intensivo y extensivo) y una zona destinada a la conservación del ecosistema.

Formulación del modelo. Los supuestos establecidos por el modelo PNLEM, respecto a las condiciones del bosque bajo manejo y las posibilidades operativas de la comunidad para las actividades de aprovechamiento son: la superficie destinada para uso forestal no cambió durante el periodo de planificación (10 años); todos los rodales son accesibles durante el periodo de planificación para realizar las actividades silvícolas prescritas.

Los tratamientos silvícolas considerados para la zona de aprovechamiento extensivo fueron: primera cosecha comercial (a realizarse en el año 1), segunda cosecha comercial (a realizarse en el año 5) y la tercera cosecha comercial (a realizarse en el año 10).

Los tratamientos silvícolas considerados para la zona de aprovechamiento intensivo fueron corta de liberación (aplicable en rodales con edades menores a 7 años), aclareo comercial ligero (aplicable en rodales con edades entre 8 y 28 años), aclareo comercial normal (aplicable en rodales con edades entre 29 y 49 años) y corta de regeneración (aplicable en rodales con edades mayores a 49 años).

Los tipos de productos maderables posibles a obtener se definieron como primarios (troza de $2,55 \mathrm{~m}$ de longitud y diámetro con corteza mayor a $25 \mathrm{~cm}$ ), secundarios (troza de $1,27 \mathrm{~m}$ de longitud y diámetro con corteza menor a $25 \mathrm{~cm}$ y mayor a $20 \mathrm{~cm}$ ) y celulósicos y otros (brazuelo de diámetro con corteza menor a $20 \mathrm{~cm}$ y mayor a $10 \mathrm{~cm}$ ).

Se consideraron 16 tipos de hábitat identificados por la comunidad, y una prioridad de conservación de cada tipo de hábitat en función de la extensión de superficie actual ocupada por el mismo dentro del bosque. Por último, para calcular el ingreso monetario presente (VP) se utilizó una tasa de interés real de $4 \%$, siendo esta constante en el horizonte de planeación. Esta se definió considerando las tasas de descuento propuestas para plantaciones forestales comerciales en México, las cuales varían entre $5 \%$ y $9 \%$ (Valdez-Lazalde y Lewis 2000, Zamudio et al. 2010). Sin embargo, considerando que el análisis corresponde a aprovechamiento de bosques naturales se decidió utilizar una tasa menor. 
Modelo matemático. Las variables de decisión del modelo son: $y_{i k}$, que toma el valor de 1 si el $i$-ésimo rodal es asignado a conservación y 0 en caso contrario (donde $i$ va desde 1 a 556); $U_{i i^{\prime}, j}$ con valor de 1 si dos rodales ( $i$ e $i^{\prime}$ ) cosechados en el $j$-ésimo periodo son colindantes y 0 en caso contrario; $P T_{i k}$, que vale 1 si el potencial de aprovechamiento del $i$-ésimo rodal (previamente definido para aprovechamiento extensivo o intensivo) coincide con la zona $k$ a la que fue asignado, donde $k=1,2,3 ; 0$ en cualquier otro caso; $F_{j}$, con valor 1 si en el $j$-ésimo periodo el volumen anual cosechado supera $150.000 \mathrm{~m}^{3}$, donde $j$ va desde 1 a 10, y 0 en cualquier otro caso.

La variable $\alpha$ es el número de hectáreas asignadas a la zona de conservación; $\alpha_{a}$ indica la superficie total determinada para conservar por tipo de hábitat $a$, donde $a$ va desde 1 a $16 ; \beta_{j}$ es al volumen maderable a cosechar en el $j$-ésimo periodo; $\beta_{j t}$ cuantifica el volumen maderable a cosechar por periodo en función del $t$-ésimo tratamiento silvícola, donde $t$ va desde 1 a $7 ; O_{q j}$ estima el volumen maderable cosechado en el periodo $j$ destinado para obtener el $q$-ésimo producto, donde $q=1,2,3$; primarios, secundarios y celulósicos y otros, respectivamente. Por último, la variable $X_{i t}$ representa el volumen maderable a cosechar del rodal $i$ en función del tratamiento silvícola $t$ en un periodo $j$; y $V_{i k t j}$ refiere al volumen maderable a cosechar del rodal $i$ perteneciente a la zona $k$, dependiendo del tratamiento silvícola $t$ asignado en el periodo $j$. El modelo se definió como sigue:

$$
\max z: C_{1}+C_{2}+C_{3}-C_{4}-C_{5}
$$

donde

$$
\begin{aligned}
& C_{1}=\sum_{j=1}^{10}\left(\frac{1000}{(1,04)^{j}}\right) \cdot \alpha \\
& C_{2}=\sum_{j=1}^{10} \cdot\left(\frac{1452}{(1,04)^{j}}\right) \cdot O_{1 j}+\sum_{j=1}^{10}\left(\frac{833}{(1,04)^{j}}\right) \cdot O_{2 j}+\sum_{j=1}^{10}\left(\frac{433}{(1,04)^{j}}\right) \cdot O_{3 j} \\
& C_{3}=(-P I n v) \cdot\left(\sum_{j=1}^{10} \sum_{i=1}^{556} U_{i i^{\prime}, j} \cdot\left(\frac{1000}{(1,04)^{j}}\right)\right) \\
& C_{4}=\sum_{i=1}^{556} P T_{i k} \cdot\left(\frac{1000}{(1,04)^{j}}\right) \\
& C_{5}=\sum_{j=1}^{10} F_{j} \cdot\left(\frac{1000}{(1,04)^{j}}\right)
\end{aligned}
$$

La función objetivo (ecuación [1]) está diseñada para maximizar el VP en el horizonte de planeación. Dicho ingreso se cuantifica por $C_{1}$, que contabiliza el beneficio económico que implica conservar el ecosistema mediante el pago de servicios ambientales (MX \$1.000/ha) (CONAFOR 2018); $C_{2}$, que refiere al ingreso generado por los tipos de productos posibles a obtener del volumen cosechado: primarios $\left(O_{1 j}\right)$, secundarios $\left(O_{2 i}\right)$ y celulósicos y otros $\left(O_{3 j}\right)$, con un precio estimado de MX $\$ 1.452 / \mathrm{m}^{3}$, MX $\$ 833 / \mathrm{m}^{3}$ y MX $\$ 433 / \mathrm{m}^{3}$, respectivamente (Parra 2016); $C_{3}$ equivale a una sanción económica (MX \$1.000/ha por no realizar la cosecha de forma dispersa en todo el bosque de producción (ausencia de actividades en ciertas zonas del bosque), considerando la probabilidad de invasión territorial (-PInv) por parte de comunidades aledañas a San Pedro El Alto, situación que ha ocurrido en las últimas décadas.

De forma similar, el término $C_{4}$ representa una penalización económica de MX \$1.000 (valor arbitrario) cada vez que un rodal sea asignado en alguna zona sin considerar su potencial de aprovechamiento; y $C_{5}$ sanciona al ingreso total con MX $\$ 1.000$ (valor arbitrario) por cada periodo en el que se cosechen más de $150.000 \mathrm{~m}^{3}$, que es el volumen definido por la comunidad como el límite máximo de cosecha por periodo.

Los términos $C_{3}, C_{4}$ y $C_{5}$ corresponden a restricciones suaves del modelo, las cuales permiten flexibilizarlo y así conseguir una solución al problema aun cuando se viole alguna o todas de las restricciones suaves. Si se incurre en alguna violación de las restricciones suaves, existe una sanción económica correspondiente que reducirá el valor obtenido por la función objetivo. Es deseable que las soluciones encontradas cumplan todas las restricciones, pero al relajar las restricciones y usar un sistema de penalización se busca encontrar soluciones cercanas al óptimo. El PNLEM está acotado por 15 restricciones. La ecuación [2] establece que la superficie total (hectáreas) destinada a conservación $(\alpha)$ deberá ser igual a la suma de las superficies destinadas al mismo propósito por cada tipo de hábitat $\left(\alpha_{\alpha}\right)$.

En la ecuación [2], $A$ representa el número de hábitats existentes en el área bajo gestión. En la ecuación [3] se definen dos componentes de $\alpha_{a}$, que son importantes para la implementación del modelo y se explican en seguida.

$$
\begin{gathered}
\alpha=\sum_{a=1}^{A} \alpha_{a} \\
\alpha_{a}=S c_{a}+S T_{a}
\end{gathered}
$$

La superficie definida por el modelo para conservación por tipo hábitat $\left(\alpha_{a}\right)$ está conformada por una primera asignación de superficies $\left(S c_{a}\right)$ por tipo de hábitat y una segunda asignación $\left(S T_{a}\right)$ que puede realizarse o no según sea el caso. Si la sumatoria de las primeras superficies asignadas $\left(S c_{a}\right)$ no iguala a la superficie deseable en conservación predefinida por el gestor forestal $(S c d)$, se generará un déficit de superficie de conservación (DDC) (ecuación [4]), el cual deberá completarse a través de un segundo compendio de superficies seleccionadas de los tipos de hábitats existentes $\left(S T_{a}\right)$ (ecuación [5]).

$$
\begin{gathered}
D D C=S c d-\sum_{a=1}^{A} S c_{a} \\
\sum_{a=1}^{A} S T_{a}=D D C
\end{gathered}
$$


El logro del complemento $S T_{a}$ solo será posible si la superficie existente de un hábitat $a\left(S E_{a}\right)$ es mayor que la primera superficie seleccionada $\left(S c_{a}\right)$, y estará acotado por $S E_{a}$ (ecuación [6]). Por lo tanto, la superficie total de conservación asignada por el modelo por tipo de hábitat será la suma de la superficie asignada en la primera etapa $\left(S c_{a}\right)$ mas la superficie asignada en la segunda etapa $(S T)$, (ecuación [3]).

$$
S T_{a} \leq S E_{a}
$$

La ecuación [7] indica que el $i$-ésimo rodal solo puede ser asignado a un tipo de zona (aprovechamiento intensivo, aprovechamiento extensivo o conservación) y esta no cambiará dentro del horizonte de planeación.

$$
\sum_{k=1}^{K} y_{i k}=1 \quad \forall i=1, \ldots, 556
$$

La ecuación [8] establece que el volumen maderable a cosechar por periodo depende del volumen cosechado en función del tratamiento silvícola aplicado.

$$
\beta_{j}=\sum_{j=1}^{J} \beta_{j t} \quad \forall t=1, \ldots, 7
$$

A su vez, este volumen se cuantificará por la suma del volumen de todos aquellos rodales programados en cada tipo de tratamiento silvícola (ecuación [9]).

$$
\beta_{j t}=\sum_{i=1}^{N} X_{i t j} \quad \forall t=1, \ldots, 7 ; j=1, \ldots, 10
$$

El volumen maderable cosechado en un rodal en un periodo $\left(V_{i k j}\right)$ será contable $\left(X_{i t j}\right)$ si y solo sí proviene de una zona que permite la cosecha $\left(Y_{i k}=\right.$ zona de aprovechamiento extensivo o intensivo) y de un tratamiento silvícola prescrito $\left(W_{i t j}\right)$ para la misma (ecuación [10]).

$$
X_{i t j}=V_{i k t j} \cdot Y_{i k} \cdot W_{i t j}
$$

La producción maderable por tipo de producto $\left(O_{q j}\right)$ en un periodo $j$, corresponde a una fracción del volumen total de cosecha determinado por el tipo de tratamiento silvícola aplicado en ese periodo (ecuación [11]). Las fracciones del volumen de cosecha $\left(D P_{q j}\right)$ que corresponden a cada tipo de producto se establecieron de acuerdo con la experiencia productiva de San Pedro El Alto y los tratamientos silvícolas definidos en el PMF de la comunidad. Así, en la tercera cosecha comercial $40 \%$ del volumen cosechado es producto primario $\left(D P_{13 j}=0,40\right)$, en la corta de liberación y en la corta de regeneración $80 \%\left(D P_{14 j}=0,80\right)$ y $70 \%$ $\left(D P_{17 j}=0,70\right)$ del volumen cosechado es producto primario, respectivamente.
Los productos secundarios se obtienen a razón de $60 \%$ del volumen extraído en la primera cosecha comercial $\left(D P_{21 j}=0,60\right), 60 \%$ del volumen extraído en la segunda cosecha comercial $\left(D P_{22 i}=0,60\right), 45 \%$ del volumen extraído en la tercera cosecha comercial $\left(D P_{23 j}=0,45\right), 15 \%$ del volumen cosechado en la corta de liberación $\left(D P_{24 j}=\right.$ $0,15), 60 \%$ del volumen cosechado en el aclareo ligero $\left(D P_{25 j}=0,60\right), 60 \%$ del volumen cosechado en aclareo normal $\left(D P_{26 j}=0,60\right)$ y $20 \%$ del volumen cosechado en la corta de regeneración $\left(D P_{27 j}=0,20\right)$.

Por último, la producción de celulósicos y otros productos se definió con las fracciones siguientes: $40 \%$ del volumen extraído en la primera cosecha comercial $\left(D P_{31 j}\right.$ $=0,40), 40 \%$ del volumen extraído en la segunda cosecha comercial $\left(D P_{32 j}=0,15\right), 15 \%$ del volumen extraído en la tercera cosecha comercial $\left(D P_{33 j}=0,15\right), 5 \%$ del volumen cosechado en la corta de liberación $\left(D P_{34 j}=0,05\right), 40 \%$ del volumen cosechado en el aclareo ligero $\left(D P_{35 j}=0,40\right), 40 \%$ del volumen cosechado en aclareo normal $\left(D P_{36 j}=0,40\right)$ y $10 \%$ del volumen cosechado en la corta de regeneración $\left(D P_{37 j}=0,10\right)$.

$$
O_{q j}=\sum_{t=1}^{T} D P_{q t} \cdot \beta_{j t} \quad \forall q=1,2,3 ; j=1, \ldots, 10
$$

La ecuación [12] restringe la cantidad mínima $\left(M_{l j}\right)$ y máxima $\left(M_{h j}\right)$ deseable de volumen a cosechar por periodo. De acuerdo con el registro de cosechas realizadas con anterioridad en la comunidad de San Pedro El Alto, el valor de $M_{l j}$ se fijó en $80.000 \mathrm{~m}^{3}$ y el valor de $M_{h j}$ en $150.000 \mathrm{~m}^{3}$.

$$
M_{l j} \leq \beta_{j} \leq M_{h j}
$$

La ecuación [13] indica el rango permitido en la intensidad de corta $\left(G_{l j}\right.$ y $G_{h j}$, respectivamente) para el volumen maderable existente $\left(V E_{i j}\right)$ del rodal $i$ en un periodo $j$, siendo este determinado por el tratamiento silvícola prescrito en cada rodal. Conforme a los tratamientos silvícolas especificados en el PFM de la comunidad, para la corta de liberación se permitió una intensidad de corta entre el 80 y $95 \%$ del volumen existente, para el aclareo comercial ligero una intensidad de corta permitida entre el 60 y $70 \%$, para el aclareo comercial normal una intensidad de corta permitida entre el 60 y $75 \%$ y para la corta de regeneración se permitió una intensidad de corta del $95 \%$. En cuanto a las intensidades de corta para los tratamientos silvícolas correspondientes a la zona de aprovechamiento extensivo, estas se definieron de forma arbitraria, ya que son actividades que no se incluyen en el PFM vigente. Para la primera cosecha comercial se permite extraer el $50 \%$ del volumen existente, para la segunda cosecha comercial el $45 \%$ y para la tercera cosecha comercial el $60 \%$.

$$
G_{l j} \cdot V E_{i j} \leq V_{i k t j} \leq G_{h j} \cdot V E_{i j}
$$


La ecuación [14] considera la capacidad mínima de procesamiento anual $\left(L I r_{j}\right)$ del aserradero de la comunidad forestal, correspondiente a $40.000 \mathrm{~m}^{3}$.

$$
L I r_{j} \leq \sum_{q=1}^{2} O_{q j}
$$

Para impedir la prescripción de dos tratamientos en el mismo rodal en un periodo $j$ y que se intervenga más de una vez el rodal $i$ en el horizonte de planeación, se formuló la ecuación [15].

$$
\sum_{k=2}^{K} \sum_{t=1}^{T} W_{i t j} \cdot Y_{i k}=1
$$

Por último, se incluye la restricción de no negatividad en el modelo (ecuación [16]).

$$
V_{i k t j} \geq 0
$$

Técnicas de solución. El PNLEM planteado se resolvió mediante tres algoritmos genéticos: uno en su versión simple (GNS), uno con mutación múltiple (GN2) y uno de mutación múltiple con elitismo (GN3). Se eligió este tipo de heurístico debido a que se ha usado con buenos resultados para resolver problemas de planificación forestal de gran dimensión que incluyen información espacial en el proceso de optimización (Fotakis et al. 2012, Pasquale 2017).

El algoritmo genético típico se define como un algoritmo de búsqueda basado en la mecánica de la selección natural y proceso de recombinación genética (Boston y Bettinger 2002). Este algoritmo parte de una población inicial aleatoria conformada por soluciones iniciales al problema (individuos). Posteriormente, la población evoluciona mediante el uso de operadores evolutivos que combinan y modifican a los individuos, creando una nueva población (conjunto de soluciones al problema).
Habitualmente se aplican tres operadores genéticos: selección, cruza y mutación. Mediante un proceso de selección algunas soluciones iniciales se definen como "padres" para su posterior reproducción, y adicionalmente se emplea un operador de mutación aleatoria que modificará la composición de las soluciones producto de la cruza o reproducción (hijos), con el fin de mejorar la diversidad en el conjunto de soluciones. Por lo tanto, sobrevivirán las soluciones más cercanas al óptimo (mejor adaptadas), y estas formarán parte del nuevo conjunto de soluciones (población) (Suesca et al. 2016). Los procesos de evaluación, selección, reproducción y mutación se repiten hasta que se alcanza el criterio de paro especificado.

En este trabajo, la técnica GNS realizó la cruza entre padres en un solo punto, seleccionado de forma aleatoria, y este indicó el punto de corte para la recombinación de los valores entre soluciones. Esta técnica también utilizó una mutación simple (solo se modifica un valor de la solución para $Y_{i k}, V_{i k j j}$ o $W_{i t j}$ ) mediante la técnica de intercambio de valores entre soluciones (swap) y considerando una probabilidad de $5 \%$ para que ocurra la mutación. En cuanto a la técnica GN2, también estableció una probabilidad de mutación de $5 \%$ y la cruza en un punto, pero el proceso de mutación fue múltiple (se modifica un valor en cada variable $Y_{i k}, V_{i k j j} \mathrm{y}$ $\left.W_{i t}\right)$, lo que permitió aumentar la diversidad de la población (soluciones). Por último, la técnica GN3, además de utilizar la cruza en un punto y la mutación múltiple con probabilidad de $5 \%$, empleó una función de mejora, en la cual, bajo un criterio elitista, selecciona un porcentaje de la población inicial conformado por las mejores soluciones encontradas y las conserva en todas las futuras generaciones. Para el caso particular, dicho porcentaje se estableció en $10 \%$. El valor de los operadores de mutación y función de mejora, se definieron de forma arbitraria, con la finalidad de balancear la diversidad en las soluciones (definida por la tasa de mutación) y la mejora de las mismas con la tasa de elitismo.

Cada algoritmo se codificó en el lenguaje de programación MATLAB ${ }^{\circledR}$ versión R2015a (The Math Works Inc. 2015) (figura 1) y se ejecutó 20 veces, realizando 18.100

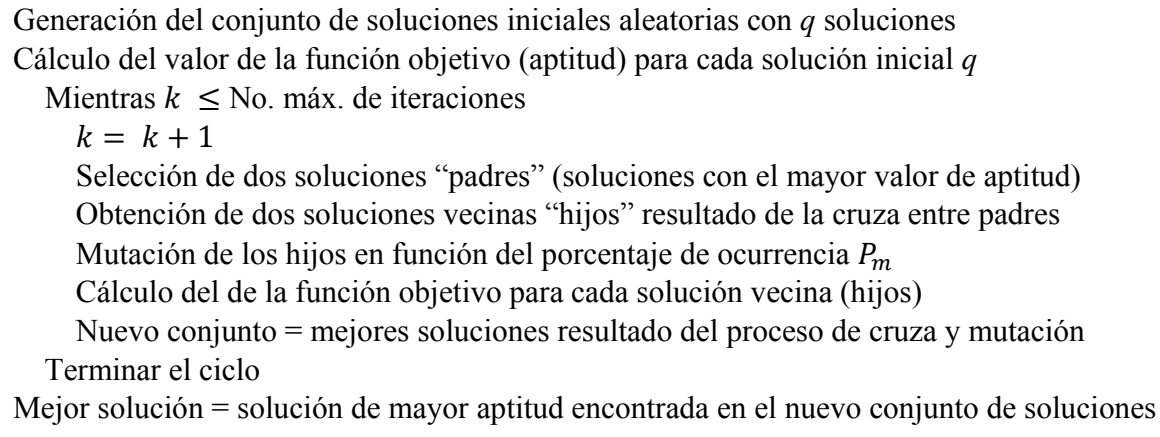

Figura 1. Pseudocódigo del algoritmo genético.

Genetic algorithm pseudocode. 
evaluaciones a la función objetivo por cada ejecución. Dicho valor se determinó empíricamente a través de pruebas experimentales, y fue seleccionado de acuerdo a su aptitud para obtener soluciones de buena calidad, es decir, que cumplieran con las restricciones del problema y encontraran el mejor valor de la función objetivo en un tiempo de cómputo razonable, siendo este aquel que puede resolver un problema de complejidad no polinomial, como es el caso de estudio, en un tiempo polinomial haciendo uso de estrategias estocásticas. Para el propósito se utilizó un ordenador con microprocesador Intel Celeron $1,60 \mathrm{GHz}$ y una memoria RAM de 8 GB.

Evaluación de soluciones del modelo. No se conoce la solución óptima al problema planteado como caso de estudio. Debido a su complejidad es difícil encontrar el óptimo en un tiempo razonable utilizando un método exacto. Por lo tanto, se consideró al mejor valor conocido como punto de referencia, el cual corresponde a un ingreso total de MX $\$ 1.000,2$ millones de pesos, estimada a partir de los datos de cosecha forestal reportados en el Programa de Manejo Forestal (PMF) vigente para la comunidad de San Pedro El Alto.

La capacidad de las técnicas heurísticas implementadas para solucionar el modelo (soluciones cercanas o mejores al valor conocido) y la confiabilidad de los resultados obtenidos se evaluó mediante el error estadístico resultante de la prueba de medianas de Bootstrap. Por otro lado, las diferencias en el desempeño de las técnicas utilizadas se determinaron mediante la prueba no paramétrica con signo de Wilcoxon. La prueba de medianas de Boostrap es una técnica no paramétrica que proporciona estimaciones del error estadístico en los intervalos de confianza, imponiendo escasas restricciones sobre las variables aleatorias analizadas (Solanas y Sierra 1992). La prueba de signos de Wilcoxon es considerada como una alternativa a la prueba de $t$ para dos muestras pareadas y el procedimiento consiste en asignar rangos a las diferencias entre pares de observaciones. De acuerdo con esta prueba, la hipótesis nula $\mathrm{H}_{0}$ menciona que, si el valor de la prueba es mayor que el valor crítico (valor en la tabla de valores críticos de Wilcoxon, correspondiente al número de datos y nivel de significancia, 52 en este caso), no existen diferencias significativas entre las comparaciones. De forma contraria la hipótesis alternativa $\mathrm{H}_{1}$ menciona que, si el valor obtenido en la prueba es menor o igual al valor crítico, estadísticamente existen diferencias significativas entre las comparaciones (Badii et al. 2012).

Adicionalmente se evaluó el tiempo computacional utilizado por cada una de las heurísticas para solucionar el modelo.

\section{RESULTADOS}

La dispersión de los valores de la función objetivo (ingreso monetario total en el horizonte de planeación) obtenidos por las tres versiones del algoritmo genético implemen- tado se presentan en un diagrama de cajas y bigotes de los ingresos obtenidos (figura 2). El algoritmo GN3 presentó la mayor dispersión, mientras que la técnica GNS presentó la menor dispersión. Aunque todas las técnicas obtuvieron soluciones lógicas acorde con el modelo, estas presentan una variación considerable en los valores obtenidos.

El valor de la mediana poblacional para el algoritmo GNS estuvo en el rango de MX $\$ 800-\$ 813$ millones de pesos y, en caso similar para el algoritmo GN3, el valor de la mediana se encontró en un rango de MX \$800-806 millones de pesos (cuadro 1). Para el caso del algoritmo GN2, el margen de error de la mediana poblacional osciló en un rango de MX $\$ 810-\$ 813$ millones de pesos (cuadro 1). Se optó por estimar la mediana, en lugar del promedio,

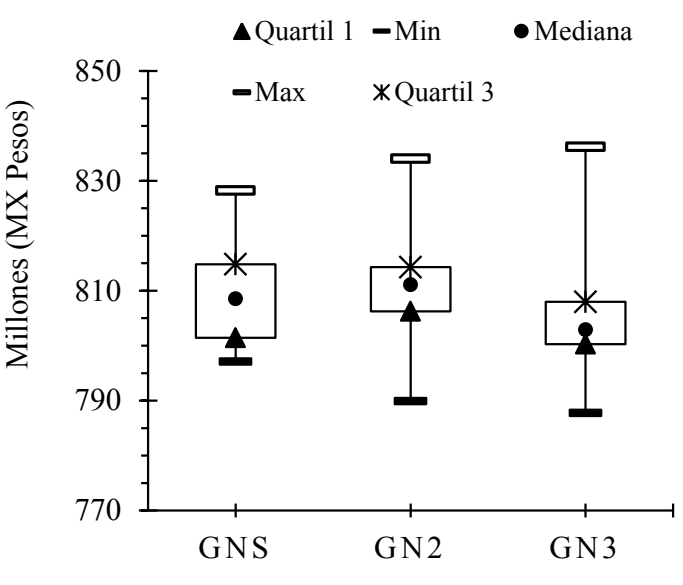

Técnicas heurísticas

Figura 2. Diagrama de cajas y bigotes de los ingresos obtenidos en el horizonte de planeación por los algoritmos genético simple (GNS), genético con mutación múltiple (GN2) y genético de mutación múltiple con elitismo (GN3).

Boxplot of the gains obtained in the planning horizon by the simple genetic algorithm (GNS), the genetic algorithm with multiple mutation (GN2) and the genetic algorithm with multiple mutation and elitism (GN3).

Cuadro 1. Resumen de la prueba de Bootstrap para los valores obtenidos por los algoritmos genéticos.

Summary of Bootstrap test for values obtained by the genetic algorithms.

\begin{tabular}{lcrc}
\hline \multicolumn{4}{c}{ Ingreso (millones de pesos) } \\
\hline & GNS & GN2 & GN3 \\
Límite superior & 812,9 & 813,3 & 806,2 \\
Límite inferior & 801,8 & 810,1 & 801,1 \\
\hline
\end{tabular}

GNS: algoritmo genético simple, GN2: algoritmo genético con mutación múltiple, GN3: algoritmo genético de mutación múltiple con elitismo. 
pues el valor de la media suele ser influenciado por valores separados del resto de los valores obtenidos (más altos o más bajos), mientras que la mediana es una métrica más adecuada para muestras pequeñas, como es el caso en este trabajo.

En cuanto a la eficiencia de las técnicas utilizadas, esta se determinó mediante el error relativo promedio $(E)$ de los valores obtenidos para la función objetivo (ingresos) con respecto al mejor valor conocido (MX \$1.000,2 millones de pesos) (ecuación [17]).

$$
E=\frac{V_{h} \cdot V_{o}}{V_{o}} \cdot 100
$$

Donde,

$V_{h}$ : valor de la solución obtenida por la técnica heurística.

$V_{o}:$ mejor valor conocido.

El error relativo de las soluciones obtenidas (ingresos) con respecto al mejor valor conocido, fue en promedio del $19,2 \%$, siendo la técnica GN3 la que presentó el error más grande con 19,54\%. Aunque la técnica GN3 obtuvo la solución que maximiza el valor de la función objetivo, esta presentó una eficiencia menor que la técnica GN2 (cuadro 2). Los resultados obtenidos para la prueba de Wilcoxon fueron de 86 para la comparación GNS vs GN2, de 74 para la comparación GNS vs GN3 y de 52 para la comparación GN2 vs GN3.

Las comparaciones GNS vs GN2 y GNS vs GN3 presentaron valores de la prueba mayores que el valor crítico, correspondiente a 52 para un total de 20 datos y un nivel de significancia de $0,05 \%$; mientras que la comparación GN2 vs GN3 presentó un valor igual al valor crítico. Por lo tanto, únicamente existió una diferencia significativa en el desempeño de las técnicas GN2 y GN3 para resolver el problema definido.

El mejor valor encontrado (máximo ingreso en el horizonte de planeación) correspondió a MX $\$ 836$ millones de pesos, mientras que el peor valor encontrado fue de MX $\$ 788$ millones de pesos, ambos valores obtenidos por la técnica GN3 (cuadro 2).
El esquema actual de manejo en San Pedro El Alto define un aprovechamiento intensivo (de acuerdo con la propuesta de zonificación) para la totalidad de la superficie bajo manejo, es decir, en las 8.427 ha consideradas en el PMF vigente. Comparativamente, la mejor solución encontrada asignó $2.227,15$ ha a la zona de conservación, 817,64 ha a la zona de aprovechamiento extensivo y $5.381,89$ ha a la zona de aprovechamiento intensivo (figura 3 ).

Esta solución estimó un ingreso total en el horizonte de planeación de MX $\$ 836$ millones de pesos como producto de los tratamientos silvícolas definidos para los aprovechamientos extensivo e intensivo. El flujo de volumen de cosecha anual se mantuvo por debajo de la meta ( 150.000 $\mathrm{m}^{3}$ anuales) en todas las anualidades, con un promedio de $132.039,29 \mathrm{~m}^{3}$ y una fluctuación promedio de $9.004,16 \mathrm{~m}^{3}$ en el horizonte de planeación (figura 4).

La fluctuación promedio del volumen de cosecha anual estipulado en el PFM de la comunidad correspondió a $15.663,32 \mathrm{~m}^{3}$ en el horizonte de planeación. A pesar de que la comunidad definió una cosecha anual de 150.000 $\mathrm{m}^{3}$, en el PMF vigente se definieron volúmenes de cosecha de $188.028,65 \mathrm{~m}^{3}$ y $160.192,53 \mathrm{~m}^{3}$ para los años 1 y 7 , respectivamente. Para los años 4 y 5 la cosecha anual programada correspondió a $116.827,15$ y $117.421,04 \mathrm{~m}^{3}$, respectivamente. Para el resto de las anualidades, el volumen de cosecha programado estuvo debajo de los $132.700 \mathrm{~m}^{3}$. Evidentemente, la técnica GN3 logró disminuir la fluctuación de volumen de cosecha anual en comparación con el plan de ordenación vigente, además de respetar el flujo de cosecha máximo definido por la comunidad.

\section{DISCUSIÓN}

El mejor ingreso estimado por el algoritmo GN3 es 16\% menor al ingreso estimado considerando el escenario actual de manejo en la comunidad de San Pedro El Alto. Sin embargo, es importante destacar que la solución de GN3 segrega del aprovechamiento silvícola $2.227,2$ ha, las cuales se destinan a conservación. En términos monetarios tal segregación representa un ingreso no percibido de MX $\$ 227,2$ millones de pesos. De manera contraria, si se adicionan los MX \$227,2 millones de pesos al ingreso obteni-

Cuadro 2. Valores obtenidos por los algoritmos genéticos en el proceso de experimentación.

Values obtained by the genetic algorithms in the experiment process.

\begin{tabular}{ccccccc}
\hline Técnica & $\begin{array}{c}\text { Mejor valor } \\
\text { conocido }\end{array}$ & $\begin{array}{c}\text { Mejor solución } \\
\text { obtenida }\end{array}$ & $\begin{array}{c}\text { Valor } \\
\text { promedio }\end{array}$ & $\begin{array}{c}\text { Peor solución } \\
\text { obtenida }\end{array}$ & Error relativo & $\begin{array}{c}\text { Tiempo de } \\
\text { ejecución (s) }\end{array}$ \\
\hline GNS & & 828,2 & 808,9 & 797,1 & 19,24 & 2.267 \\
GN2 & $1.000,2$ & 834,0 & 810,9 & 789,9 & 19,03 & 2.625 \\
GN3 & & 836,2 & 805,4 & 787,7 & 19,59 & 2.346 \\
\hline
\end{tabular}

GNS: algoritmo genético simple, GN2: algoritmo genético con mutación múltiple, GN3: algoritmo genético de mutación múltiple con elitismo. 


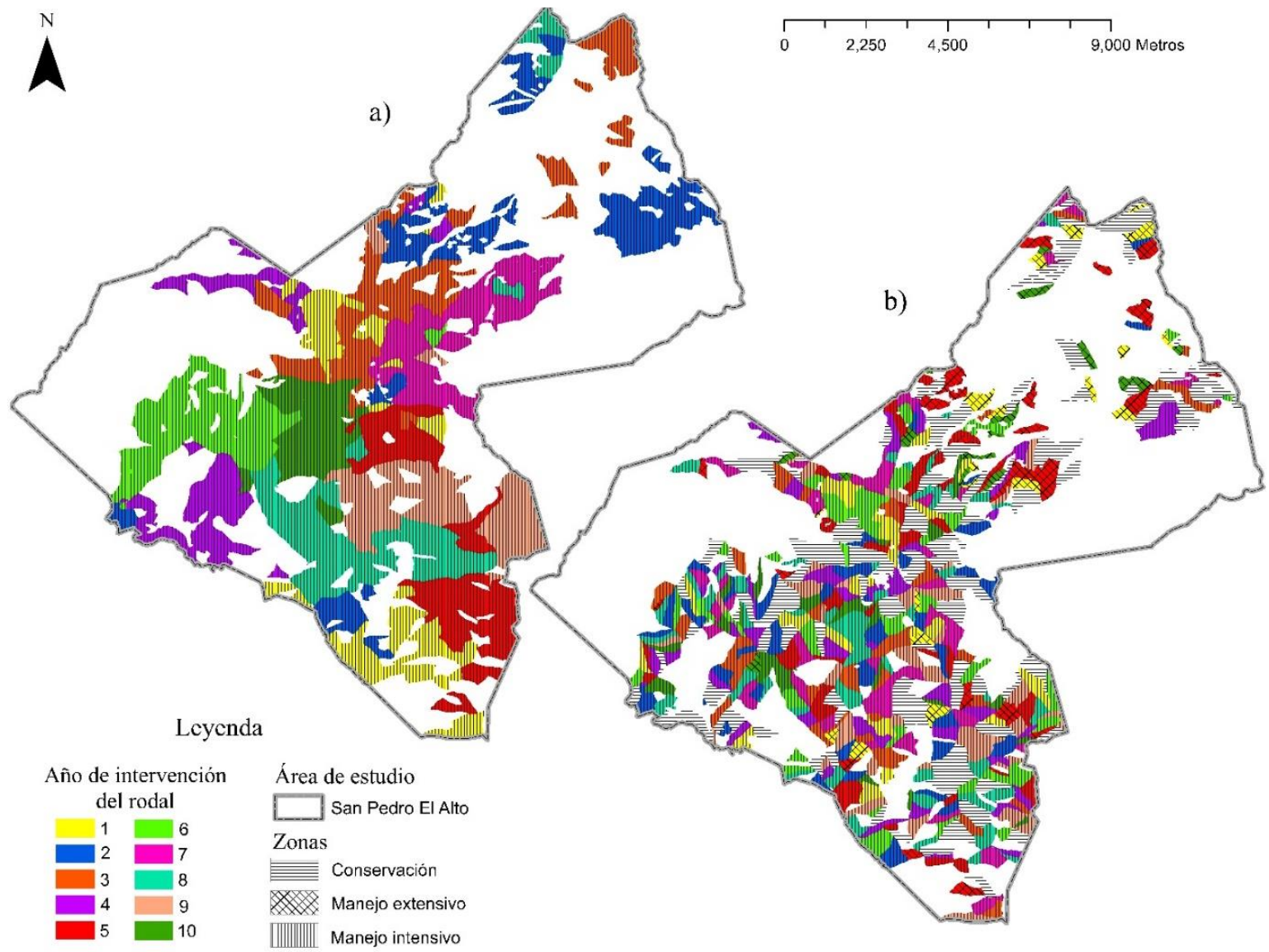

Figura 3. Distribución de las anualidades de cosecha y zonas TRIAD para a) escenario actual del PMF vigente de San Pedro El Alto y b) mejor solución obtenida por el algoritmo genético de mutación múltiple con elitismo (GN3).

Annual harvest distribution and TRIAD zones for A) current scenario of the PMF in San Pedro El Alto and B) best-found solution obtained by the genetic algorithm with multiple mutation and elitism (GN3).

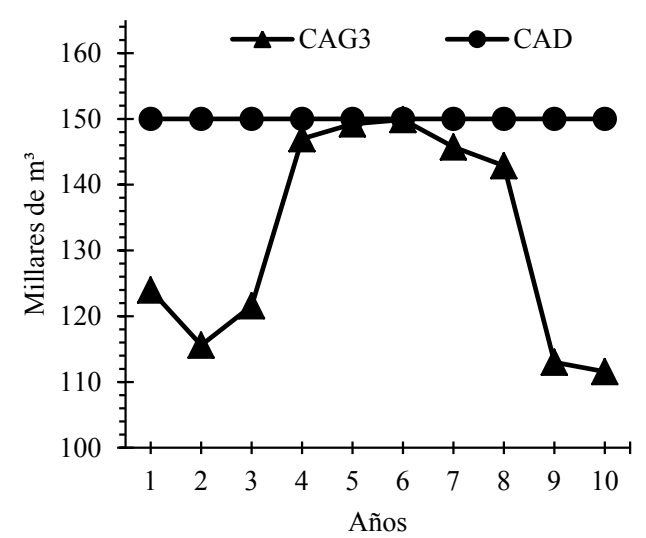

Figura 4. Flujo del volumen de cosecha anual estimado (CAG3) por el algoritmo genético de mutación múltiple con elitismo (GN3), comparado con el flujo de cosecha anual definido como deseable por la comunidad de San Pedro El Alto (CAD).

Estimated annual harvest flow (CA3) by the genetic algorithm with multiple mutation and elitism (GN3), compared to annual harvest flow defined by the community of San Pedro El Alto (CAD). do por la técnica GN3 (MX \$836 millones de pesos), fácilmente se supera la mejor solución conocida. No excluir del aprovechamiento la superficie definida como conservación implicaría un ingreso promedio de MX $\$ 10.202,6 / \mathrm{ha} \mathrm{com-}$ parado con MX $\$ 1.000 /$ ha que se definieron como ingreso potencial para áreas en conservación.

Lo anterior es congruente con lo reportado por Mönkkönen et al. (2014), quienes mencionan que las acciones de conservación, a menudo, restringen otras actividades productivas y generan costos que deben descontarse del ingreso total. Idealmente, dicho costo debería de cubrirse con la comercialización de bienes y servicios ambientales, pero debido a que actualmente no existe un mercado definido con tal fin, la valoración económica de estos bienes y servicios no puede ser determinada directamente (Hauer et al. 2010).

Respecto al desempeño de los algoritmos genéticos, de acuerdo con Pukkala y Heinonen (2006), el principal problema de estos es que su velocidad y rendimiento dependen, en gran medida, de los parámetros guía en el proceso de búsqueda. Para el problema planteado, la comparación 
del tiempo computacional requerido entre la mejor solución conocida y la mejor obtenida, no puede realizarse de manera precisa, pues no se conoce la solución óptima al mismo; pero si existe una diferencia marcada entre la eficiencia de las técnicas utilizadas que resuelven el modelo en aproximadamente 40 minutos y el tiempo invertido por la comunidad para obtener la mejor solución conocida (PMF vigente), siendo este de aproximadamente tres meses.

La razón de encontrar una diferencia poco significativa en el desempeño de los algoritmos puede atribuirse al número de soluciones obtenidas para cada técnica utilizada, ya que determinar el número de veces que el algoritmo debe ejecutarse es un elemento crítico para obtener una mejor solución. No obstante, la técnica GN3 fue capaz de obtener mejores soluciones gracias a su criterio elitista, el cual conforma a las nuevas poblaciones sin empeorar la calidad de las soluciones (Coello 1995, Alancay et al. 2016).

Por otro lado, la capacidad de resolución del problema demostrada por los algoritmos genéticos utilizados coincide con la descrita por Pasquale (2017), quien menciona que estos algoritmos son técnicas poderosas para resolver modelos de grandes dimensiones con muchas variables o restricciones, para los cuales la búsqueda de una solución óptima es demasiado costosa. Sin embargo, es necesario aclarar que debido a que cada situación es única, el rendimiento de las técnicas heurísticas dependerá de su diseño y los parámetros utilizados para el problema definido.

Para el caso de estudio los supuestos y parámetros del modelo fueron definidos en conjunto por el panel de expertos y la comunidad de San Pedro El Alto, de acuerdo a las condiciones propias del bosque, a los criterios de operación y a las metas de producción establecidas por la comunidad. En cuanto a los parámetros utilizados por las técnicas heurísticas, es necesario precisar que la falta de análisis de sensibilidad para estos valores críticos, representa una limitante en los resultados obtenidos y en el alcance del modelo.

Es importante mencionar que, aunque el enfoque TRIAD representa una alternativa para asignar diversos objetivos forestales en áreas separadas y definir estrategias de manejo independientes, este no permite definir un estándar respecto a la superficie ideal a asignar en cada zona. La asignación del territorio a una u otra zona estará definida por la calidad y cantidad de beneficios obtenidos en cada una, así como por el conjunto de objetivos de gestión determinados por los propietarios de los recursos forestales.

Idealmente este tipo de planificación debe realizarse para masas forestales que aún no están sujetas a un plan de manejo y de las cuales se pretende obtener múltiples productos como madera y productos forestales no maderables, así como mantener la biodiversidad y otros servicios que proveen los ecosistemas forestales. Sin embargo, la calidad de los resultados obtenidos por el modelo y las técnicas heurísticas implementadas son comparables a la calidad de los resultados obtenidos por los expertos al realizar el PMF de la comunidad de San Pedro El Alto. La princi- pal ventaja del algoritmo con mejor desempeño (GN3) no radica en el aumento del valor de la función objetivo, sino en la posibilidad de generar de manera eficiente, a través del enfoque TRIAD, un conjunto de escenarios de manejo forestal suficientemente buenos para fines prácticos y sin duda muchos de ellos mejores a los que se podrían obtener por ensayo y error.

\section{CONCLUSIONES}

El modelo matemático desarrollado, conjuntamente con el algoritmo de solución implementado, constituyen una herramienta para facilitar el proceso de análisis para la planificación del aprovechamiento del bosque, al reducir el tiempo requerido para generar o solucionar un escenario de gestión forestal de interés para el dueño o el administrador. Más relevante, permite optimizar el ingreso producto de la cosecha forestal mientras se cumple con restricciones de conservación, situación no aplicada comúnmente en los planes de manejo forestal en México.

La validación del modelo por parte de expertos en el área y la comunidad de San Pedro El Alto, confirma la capacidad de este para ser replicado bajo diferentes escenarios de producción y condiciones del recurso forestal, equilibrando las demandas de la industria con otros valores ecológicos como la conservación del ecosistema, y así propiciar el mantenimiento a futuro de la variedad de bienes y servicios que los recursos forestales proporcionan.

Los algoritmos genéticos implementados en este trabajo, para solucionar el modelo matemático planteado, ofrecen de manera eficiente soluciones de calidad aceptable a un problema complejo de optimización real. Sin embargo, se debe tener en cuenta que la capacidad de resolución de cada algoritmo es única debido a las diferentes estrategias utilizadas para definir el tamaño de la población, la función de aptitud y la ejecución de los operadores genéticos.

\section{AGRADECIMIENTOS}

Los autores agradecen la disposición siempre diligente de la comunidad de San Pedro El Alto, Oaxaca para compartir datos e información de su predio, particularmente la apertura del Ing. Raúl M. Hernández Cortez, asesor técnico de la comunidad.

\section{REFERENCIAS}

Alancay N, S Villagra, A Villagra. 2016. Algoritmos metaheurísticos trayectoriales para optimizar problemas combinatorios. Informes-Cientificos 8(3): 56-75. DOI: http://dx.doi. org/10.22305/ict-unpa.v8i3.222

Badii MH, A Guillen, LA Araiza, E Cerna, J Valenzuela, J Landeros. 2012. Métodos no-paramétricos de uso común. Daena: International Journal of Good Conscience 7(1): 132-155.

Boston K, P Bettinger. 2002. Combining tabu search and genetic algorithm heuristic techniques to solve spatial harvest scheduling problems. Forest Science 48(1): 35-46. DOI: https:// 
doi.org/10.1093/forestscience/48.1.35

Bouchard M, S D’Amours, M Rönnqvist, R Azouzi, E Gunn. 2017. Integrated optimization of strategic and tactical planning decision in forestry. European Journal of Operational Research 259(3): 1132-1143. DOI: https://doi. org/10.1016/j.ejor.2016.11.022

Coello CA. 1995. Introducción a los algoritmos genéticos, soluciones avanzadas. Tecnologías de Información y Estrategias de Negocios 3(17): 5-11.

CONAFOR (Comisión Nacional Forestal, MX). 2018. Reglas de Operación Programa Apoyos para el Desarrollo Forestal Sustentable 2018. Consultado 22 jun. 2018. Disponible en https:/www.gob.mx/conafor/es/documentos/reglas-deoperacion-2018

Côté P, R Tittler, C Messier, DD Kneeshaw, A Fall, MJ Fortin. 2010. Comparing different forest zoning options for landscape-scale management of the boreal forest: possible benefits of the TRIAD. Forest Ecology and Management 259(3): 418-427. DOI: https://doi.org/10.1016/j.foreco.2009.10.038

Fotakis DG, E Sidiropoulos, D Myronidis, K Ioannou. 2012. Spatial genetic algorithm for multi-objective forest planning. Forest Policy and Economics 21(1): 12-19. DOI: https://doi.org/10.1016/j.forpol.2012.04.002

Hauer G, S Cumming, F Schmiegelow, W Adamowicz, M Weber, R Jagodzinski. 2010. Tradeoffs between forestry resource and conservation values under alternate policy regimes: a spatial analysis of the western Canadian boreal plains. Ecological Modelling 221(21): 2590-2603. DOI: https://doi. org/10.1016/j.ecolmodel.2010.07.013

Hof J, M Bevers, L Joyce, B Kent. 1994. An integer programming approach for spatially and temporally optimizing wildlife populations. Forest Science 40(1): 177-191. DOI: https://doi.org/10.1093/forestscience/40.1.177

Hoganson HM, NG Meyer. 2015. Constrained optimization for addressing forest-wide timber production. Canadian Journal of Forest Research 1(1): 33-43. DOI: 10.1007/s40725015-0004-x

Marušák, R., J. Kašpar, R. Hlavatý, R. Kotek, K, Kuželka y P. Vopěnka. 2015. Alternative modelling approach to spatial harvest scheduling with respect to fragmentation of forest ecosystem. Environmental Management 56(5): 1134-1147. DOI: $10.1007 / \mathrm{s} 00267-015-0558-1$

Mönkkönen M, A Juutinen, A Mazziotta, K Miettinen, D Podkopaev, P Reunanen, H Salminen, OP Tikkanen. 2014. Spatially dynamic forest management to sustain biodiversity and economic returns. Journal of Environmental Management 134(1): 80-89. DOI: https://doi.org/10.1016/j.jenv$\underline{\operatorname{man} .2013 .12 .021}$

Murray AT, RL Church. 1995. Heuristic solution approaches to operational forest planning problems. Operational Research- Spectrum 17(2-3): 193-203. DOI: https://doi. org/10.1007/BF01719265

Parra JP. 2016. Crecimiento y evaluación financiera para plantaciones de Pinus patula Schiede. ex Schltdl. et Cham. en Zacualpan, Veracruz. Tesis Maestro en Ciencias Forestales. Estado de México, México. Posgrado en Ciencias Forestales, Colegio de Postgraduados. $127 \mathrm{p}$.

Pasquale S. 2017. Parallel genetic algorithm in the cloud. Tesis Doctor en Ciencias. Salerno, Italia. Departamento de Ciencias Empresariales, Universidad de Salerno. 150 p.

Pukkala T, T Heinonen. 2006. Optimizing Heuristic Search in forest planning. Nonlinear Analysis: Real World Applications 7(5): 1248-1297. DOI: https://doi.org/10.1016/j.nonrwa.2005.11.011

Quintero MA, M Jerez, M Ablan. 2010. Métodos heurísticos en la planificación del manejo forestal: un ejemplo de aplicación. Revista Forestal Venezolana 54(2): 183-194.

Suesca E, M Bermúdez, C Mejia, LA Cobo, L Rodríguez. 2016. Diseño de celdas de manufactura considerando el balanceo de las cargas de trabajo con algoritmos genéticos. Revista ONTARE 4(1): 89-122. DOI: https://doi. org/10.21158/23823399.v4.n1.2016.1517

Seymour RS, ML Hunter. 1992. New forestry in eastern spruce-fir forests: principles and applications to Maine. Orono, Maine. University of Maine. 716 p.

Solanas A, V Sierra. 1992. Bootstrap: fundamentos e introducción a sus aplicaciones. Anuario de Psicología 55(4): 143154.

The MathWorks Inc. 2015. MATLAB The language of technical computing. Language reference manual. Consultado 10 ene. 2019. Disponible en https://www.mathworks.com/ help/matlab/release-notes-R2015a.html

Tittler R, E Filotas, J Kroese, C Messier. 2015. Maximizing conservation and production with intensive forest management: it's all about location. Environmental Management 56(5):1104-1117. DOI: $10.1007 / \mathrm{s} 00267-015-0556-3$

Valdez-Lazalde JR, DK Lewis. 2000. Economics of thinning alternatives applied to Patula pine stands in Puebla, México. Agrociencia 34(6): 759-771. Disponible en https://www. colpos.mx/agrocien/Bimestral/2000/nov-dic/art-11.pdf

Vergara FP, CD Palma, H Sepúlveda. 2015. A comparison of optimization models for lumber production planning. Bosque 36(2): 239-246. DOI: 10.4067 / S0717$\underline{92002015000200009}$

Zamudio FJ, JL Romo, OA Cervantes. 2010. Evaluación financiera y de riesgo de una plantación forestal comercial en Zihuateutla, Puebla. Revista Chapingo Serie Ciencias Forestales y del Ambiente 16(1): 69-78. DOI: http://dx.doi. org/10.5154/r.rchscfa.2009.05.012 